DOI: http://dx.doi.org/10.15359/rgac.1-56.6

Revista Geográfica de América Central. No 56 ISSN 1011-484X, enero-junio 2016

pp. 137-161

\title{
DIMENSIÓN GEOGRÁFICA DE LOS LUGARES DE SOCIALIZACIÓN ENTRE HSH EN LA CIUDAD DE GUATEMALA Y SU VINCULACIÓN CON EL TURISMO
}

\author{
GEOGRAPHICAL DIMENSION OF MSM \\ SOCIALIZATION SPACES IN GUATEMALA CITY AND \\ ITS RELATIONSHIP WITH TOURISM
}

\author{
Álvaro Sánchez-Crispin ${ }^{1}$ \\ Gerardo Mollinedo-Beltrán ${ }^{2}$ \\ Universidad Nacional Autónoma de México
}

\section{RESUMEN}

Este trabajo tiene como objetivo explicar, territorialmente, los sitios de socialización entre hombres que tienen sexo con otros hombres (HSH) en la Ciudad de Guatemala y su relación con la actividad turística. En una primera parte, se exponen algunos de los conceptos centrales referentes al estudio de los lugares de encuentro de este segmento de la población, desde una perspectiva geográfica. Enseguida, se presenta una visión general de la capital guatemalteca como lugar central del país y nodo principal de servicios para comunidades allende su demarcación nacional, en términos económicos en general y turísticos en especial. En la tercera parte del texto se exponen los resultados de investigación que revelan la expresión territorial de los lugares estudiados. Un hallazgo de este trabajo indica que aunque el volumen demográfico de esta ciudad es considerable, dispone de pocos espacios de socialización de este tipo; sin embargo, su hinterland se expande hacia distintos sitios del país y de naciones vecinas, como El Salvador.

\footnotetext{
Doctor (Universidad de Londres). Instituto de Geografía, UNAM. Correo electrónico: asc@igg.unam.mx

2 Maestro (Universidad Nacional Autónoma de México). Posgrado en Geografía, UNAM. Correo electrónico: gemobe2001@comunidad.unam.mx
} 
Álvaro Sánchez-Crispín, Gerardo Mollinedo-Beltrán. Geographical dimension of

MSM socialization spaces in Guatemala City and its relationship with tourism

DOI: http://dx.doi.org/10.15359/rgac.1-56.6

Palabras clave: Ciudad de Guatemala, turismo, HSH

\begin{abstract}
This paper examines the issue of the territorial arrangement of MSM (men who have sex with men) socialization spaces in Guatemala City and its relationship with tourism. First, we analyze some of the main concepts associated with the explanation of how such places have managed to occupy certain areas within the urban area. Then, we present an overview of Guatemala City as the central place of the country providing services to nationals and foreigners alike. The main research results are given in the last part of the paper, pinpointing at the territorial expression of the places studied. One important finding is that, regardless of its demographic volume, Guatemala City offers a limited number of homoerotic locations. These have a clearly identifiable hinterland, drawn along the southern areas of the country, and including places in neighboring countries such as El Salvador.
\end{abstract}

Keywords: Guatemala City, tourism, MSM

\title{
Introducción
}

Los lugares de socialización gay y de HSH (hombres que tienen sexo con otros hombres) han sido objeto de estudio a partir de perspectivas sociológicas, antropológicas y geográficas, en años recientes. La Geografía es una plataforma adecuada sobre la que se puede desarrollar este tipo de trabajo investigativo. Para el caso de México, la obra de López y van Broeck (2013) marca un hito, entre otras razones, porque se constituye de estudios sobre distintas ciudades de México, varias de ellas con importante actividad turística, en los que una preocupación principal es explicar la configuración territorial de lugares de socialización de $\mathrm{HSH}$ al interior de esos espacios urbanos. Una concreción científica importante, derivada de este contexto, es de carácter cartográfico: los mapas sintéticos en los que se representan los hallazgos relevantes de cada investigación. En consistencia con esto, este trabajo se enfoca a examinar la territorialidad de los espacios de socialización entre HSH en la capital guatemalteca, algunos de los cuales están vinculados con un cierto grado de actividad turística.

Interesa aquí examinar la localización y densidad de estos sitios al interior de la capital guatemalteca, como indicativos de la existencia de servicios en atención al ocio (cf. Santos, 2006). Se parte de la idea de que, al interior de esta urbe, hay un patrón de concentración en el Centro Histórico, sin una densificación notoria de esos lugares, y que esto conforma un núcleo de atracción al que llegan $\mathrm{HSH}$, bien porque tienen que realizar diversas actividades en el centro de la ciudad y aprovechan su tránsito para visitar esos lugares, o porque específicamente viajan hasta allá para socializar. 


\section{Marco teórico-conceptual}

Diversos autores han publicado trabajos acerca de los espacios de socialización de HSH en ciudades de América Latina, como Santiago de Chile (Carrasco, 2005), Quito (Mancero, 2007), Monterrey (López y Sánchez, 2004) y la Ciudad de México (Laguarda, 2011). En el caso de esta última, Tripp (1975) arguye que, en los años setenta, existía sólo un bar gay y que era muy difícil que la población local entrara sin trabazón a ese sitio, por miedo a ser descubierto; en cambio, los turistas (en general estadounidenses) que sabían de ese establecimiento (seguramente el autor alude al bar L'Hardys; cf. Sánchez y López, 1997) lo frecuentaban sin mayor trámite pues no se encontraban en su lugar habitual de residencia. Ese autor esgrime argumentos semejantes para sitios ubicados en San Juan de Puerto Rico, en esos mismos años. Con el paso del tiempo y diversos acontecimientos de orden mundial, como los movimientos de liberación (Stonewall es uno de ellos), en las grandes ciudades del mundo y América Latina, los HSH, como parte de la comunidad diverso-sexual, se apoderaron de sitios de socialización urbana, como bares, restaurantes, discotecas y saunas, cada vez en mayor número.

En los últimos dos decenios, la subcultura gay, revolucionaria en un principio, se ha integrado a los circuitos de producción y consumo del capitalismo, en general en lo que tiene que ver con la provisión de servicios $\mathrm{y}$, en particular, con el turismo y el ocio (Ibid.). Esto es cierto para países de América Latina, en cuyas ciudades más pobladas existen diversos negocios dirigidos a una clientela de $\mathrm{HSH}$; sin embargo, esta integración económica de la subcultura gay no ha significado, necesariamente, integración social. En apariencia, en tanto haya más progreso en una ciudad (al menos en las sociedades occidentales, pobres o ricas), más apertura habría para instalar negocios dirigidos a HSH y, en consecuencia, más tolerancia hacia éstos. Buenos Aires, Sao Paulo, Lima y la Ciudad de México disponen de una oferta de negocios y servicios variada, con hinterlands amplios, para HSH. Aún así, en esas ciudades todavía prevalecen algunas conductas de intolerancia hacia los HSH. Para el caso de la Ciudad de Guatemala, una investigación hecha sobre hombres gays que frecuentan el Parque Central (sitio conocido de socialización gay), indica que el 80\% de los entrevistados fue rechazado por su familia una vez que reveló su preferencia sexual; también afirmaron sentirse discriminados al buscar un 
Álvaro Sánchez-Crispín, Gerardo Mollinedo-Beltrán. Geographical dimension of

MSM socialization spaces in Guatemala City and its relationship with tourism

DOI: http://dx.doi.org/10.15359/rgac.1-56.6

empleo o solicitar un servicio médico, y refirieron tener miedo a ser agredidos física y psicológicamente por su condición sexual (Obando, 2003).

La construcción de identidades sexuales se negocia en lo que Hubbard (citado por Santos, 2006) denomina lugares de encuentro, que pueden tener distintos grados de visibilidad geográfica en la trama urbana: desde barrios concretos como Chueca, en Madrid, hasta sitios dispersos, casi invisibles, en la trama urbana de Lima (Arboleda, 1995). La movilidad de los HSH hacia lugares de ocio nocturno (y diurno, cada vez más) es fluida y las distancias por recorrer no son un obstáculo para ello. La espacialización de la experiencia gay es crucial en la formación de la identidad gay (Santos, op. cit.). Este proceso se refiere a los desplazamientos a parques, baños (retretes), saunas y cines ubicados en ciudades de considerable tamaño demográfico, como la capital guatemalteca, realizados por quienes no quieren ser reconocidos, pero que los visitan, en forma incesante; muchos de ellos viven en barrios de la periferia urbana o en ciudades pequeñas de la zona metropolitana de la Ciudad de Guatemala. Los HSH que, por distintos motivos de visitación, se encuentran en esa ciudad, como turistas, descubren complacencia en este contexto.

\section{Área de estudio: la Ciudad de Guatemala y el turismo}

Fundada hacia 1775, en un sitio llamado La Ermita (Gellert, 1990), la zona metropolitana de la Ciudad de Guatemala es la más poblada de América Central, con más de 4 millones de habitantes (Gobierno de Guatemala, 2014). Su Centro Histórico tiene una traza colonial, con una forma cuadrada casi perfecta (Caplow, 1949) que, sucesivamente, se ha desdibujado por motivos del crecimiento propio de la ciudad o por fenómenos naturales como los sismos, y de desplazamientos de población durante la guerra civil de los años setenta y ochenta. Esta ciudad posee infraestructura de transporte y alojamiento regional de primer orden que también sostiene una actividad turística importante (cf. Sánchez-Crispín, Mollinedo y Propin, 2011; Sánchez-Crispín y Propin, 2010); cuenta con la mayor cantidad de hoteles de distintas categorías ( $15 \%$ del total existente en el país), en especial de 4 y 5 estrellas, que difícilmente se encuentran en otros lugares del territorio nacional; tiene centros comerciales muy grandes y provee de servicios para atender las necesidades de turistas nacionales y extranjeros, como bancos y agencias de viaje (Cicerone, 2012). Estas características 
Álvaro Sánchez-Crispin, Gerardo Mollinedo-Beltrán. Dimensión geográfica de los lugares de socialización entre HSH en la Ciudad de Guatemala y su vinculación con el turismo

DOI: http://dx.doi.org/10.15359/rgac.1-56.6

de equipamiento e infraestructura se asocian con el tipo de turismo predominante en la ciudad, el de negocios, y facilitan la estancia de hombres extranjeros, aunque sea por una noche, algunos de los cuales pueden estar interesados en visitar sitios de socialización entre HSH.

La ciudad está dividida en 25 zonas, entre las que existen rasgos de segregación marcados, tanto por la capacidad adquisitiva de quienes viven en ellas como por la presencia diferenciada de comercios y oferta de servicios (Cifuentes, 2008). El Centro Histórico está contenido en la Zona 1 y cuenta con la mayor cantidad de recursos culturales para el turismo (iglesias, plazas, museos). Sin embargo, los turistas extranjeros no se alojan en esta zona por razones, básicamente, de seguridad. Algunos de los inmuebles de las manzanas originales del centro de la ciudad (de un solo nivel y con patios interiores) han sido convertidos y ocupados por negocios de diversa índole, entre ellos, los orientados a atender una clientela de HSH. La zona central de la ciudad, sirve como lugar de transbordo de diferentes tipos de transporte (taxis, Transmetro, colectivos), lo que facilita la llegada de personas de distintos puntos de la zona metropolitana; y es el núcleo articulador de festividades y celebraciones, como las de Semana Santa, que atraen una cantidad notoria de visitantes. Las zonas 4, 9 y 10 (ésta llamada también Zona Viva) se encuentran entre las más ricas y consolidadas de la trama urbana; en ellas se aloja una proporción importante de turistas internacionales que visitan Guatemala, por la provisión de servicios hoteleros de primera categoría y la facilidad para acceder a centros comerciales tipo mall; ahí, los lugares de socialización entre HSH son pocos. Se registra, así, una separación espacial entre estos, ubicados en su mayoría en la Zona 1, y las áreas preferidas por los turistas internacionales, que se ahonda si se considera que los transportes intraurbanos no conectan con fluidez al Centro Histórico con estas zonas.

La Ciudad de Guatemala es el punto convergente de la infraestructura de comunicaciones terrestres y aéreas del país. La mayor proporción de los turistas extranjeros, provenientes de los principales mercados emisores (El Salvador, Honduras, Estados Unidos, Canadá y México) arriban por el aeropuerto de La Aurora, de esa ciudad (SITCA, 2013). Quienes ahí pernoctan, encuentran problemas de movilización intraurbana, inseguridad en muchas de las zonas de esta ciudad, oferta hotelera de clase superior en el Centro Histórico y la relativa lejanía entre este y la Zona 10, 
Álvaro Sánchez-Crispín, Gerardo Mollinedo-Beltrán. Geographical dimension of

MSM socialization spaces in Guatemala City and its relationship with tourism

DOI: http://dx.doi.org/10.15359/rgac.1-56.6

donde se ubican los mejores hoteles, centros comerciales y servicios para la actividad turística, cuya clientela es, en una proporción significativa, internacional. Aunque la ciudad contiene y recibe una parte importante del turismo de negocios que llega al país, en realidad la economía turística guatemalteca está más asociada con el turismo cultural de cosmovisión maya, de aventura y ecoturismo.

\section{Marco metodológico}

Este trabajo se relaciona con y toma parte del procedimiento metodológico de otros, sobre la misma línea de investigación, hechos por Levine (1979), Guasch (1995), López y Sánchez (op. cit.) y López y van der Broeck (op.cit.). En el presente estudio, se persigue revelar la territorialidad de los lugares de socialización entre HSH en la Ciudad de Guatemala y asociarla con el turismo. Con este fin, se hicieron cinco viajes, de distinta duración, a la capital guatemalteca, entre enero de 2010 y abril de 2015 , cuando se procedió a completar dos fases indagatorias, de ejecución simultánea. Una requirió la realización de entrevistas no estructuradas, a actores clave, asociados con la dinámica turística y los lugares de socialización gay y entre HSH de esta ciudad, como choferes de taxis, empresarios del sector, transeúntes de la Sexta Avenida en el centro de la ciudad, empleados de hoteles y proveedores de servicios personales, entre otros. Este procedimiento fue fundamental, pues no se dispone de guías impresas sobre estos lugares de la Ciudad de Guatemala; más aún, algunas de las fuentes primarias de información, al respecto, son sitios de internet (blogs) o por viva voz, en la calle o sitios de socialización a puerta cerrada. Lo obtenido en esta fase, permitió la descripción y explicación de los lugares que se describen líneas abajo. Asimismo, se verificó la existencia, funcionamiento y articulación de estos sitios en la trama urbana, y se les asoció con la impronta actual del turismo en la capital guatemalteca, en razón de su proximidad a puntos de interés turístico.

La representación cartográfica se basó en la consideración de la metodología de tipificación probabilística; así se obtuvo una clasificación de sitios por tipo que permitió el agrupamiento territorial a partir de la cualificación y ponderación de cuatro variables que caracterizan los sitios de socialización entre HSH (ver el procedimiento metodológico en Propín, 2003). Se consideraron las variables cualitativas referentes a antigüedad, 
costo de entrada, promoción y calidad de las instalaciones; a cada una se le asignó un valor jerárquico arbitrario (alto, medio, bajo), cuya combinación y organización en nubes tipológicas generó los cuatro tipos de lugares indicados en el mapa correspondiente (figura 1). Se revela así, su arreglo territorial en el área urbana y se les presenta enseguida con una lógica de secuenciación basada en las zonas postales en las que se divide administrativamente la ciudad de Guatemala.

\section{Resultados: lugares de socialización entre HSH en la Ciudad de Guatemala}

Se detectaron 33 lugares de socialización entre HSH en esta ciudad, la mayoría ubicados en el Centro Histórico. Esta situación debe ser contextualizada en un ambiente en el que casi ninguno es visible para la población local o los turistas que visitan esta urbe. No hay banderas arco iris ni letreros que evidencien la existencia de estos espacios, su accesibilidad es, frecuentemente, complicada y hay que recurrir a informantes locales para poder hallarlos. 
Álvaro Sánchez-Crispín, Gerardo Mollinedo-Beltrán. Geographical dimension of MSM socialization spaces in Guatemala City and its relationship with tourism

DOI: http://dx.doi.org/10.15359/rgac.1-56.6

Figura 1. Ciudad de Guatemala: distribución territorial de los lugares de socialización de HSH

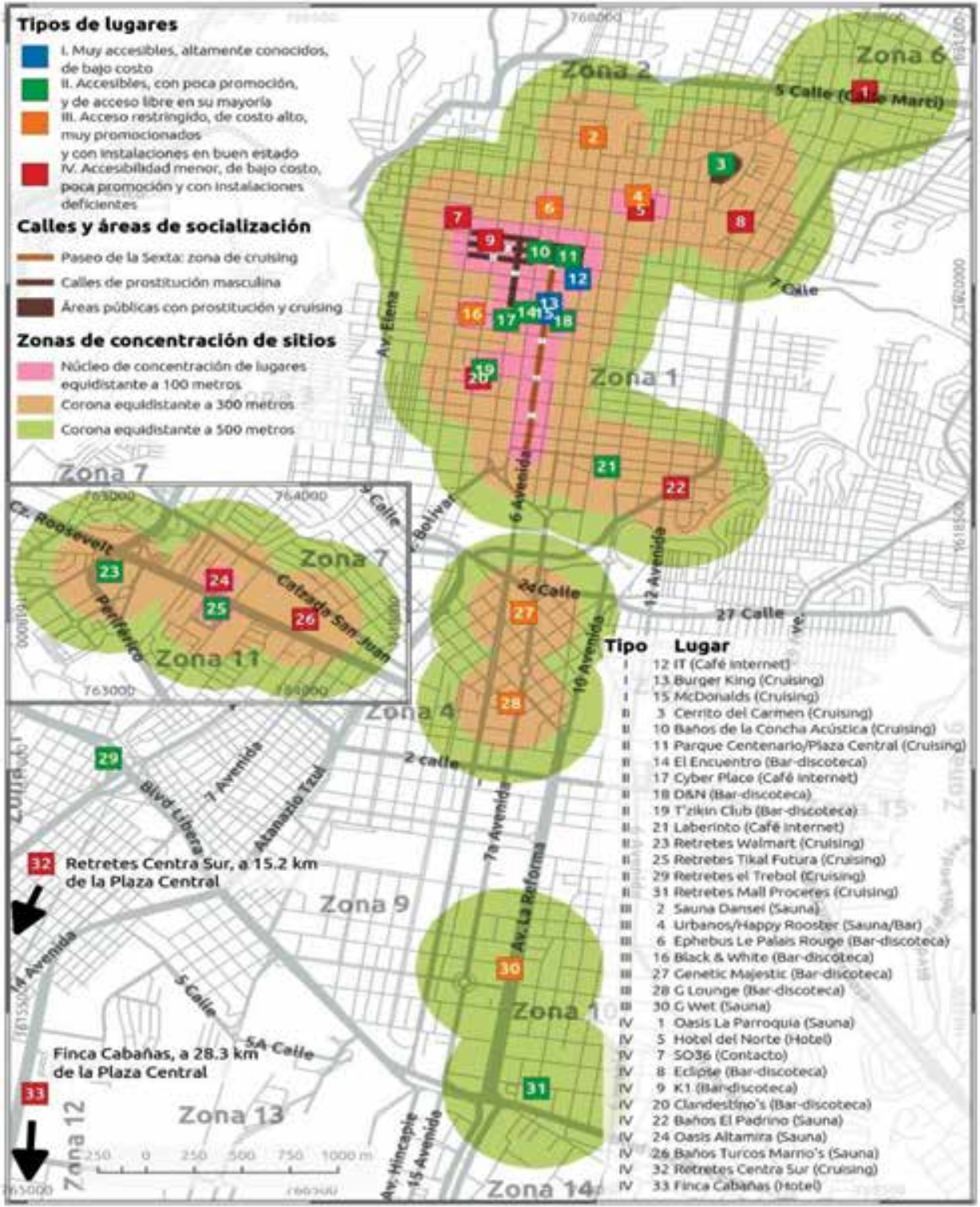

Fuente: elaborado con base en los trabajos de campo realizados entre 2010 y 2015 
Álvaro Sánchez-Crispín, Gerardo Mollinedo-Beltrán. Dimensión geográfica de los lugares de socialización entre HSH en la Ciudad de Guatemala y su vinculación con el turismo

DOI: http://dx.doi.org/10.15359/rgac.1-56.6

Zona 1. La característica fundamental de los lugares hallados es su relativa dispersión geográfica dentro del propio Centro Histórico de la Ciudad de Guatemala (figura 1). A pesar de que las distancias que median entre ellos están por debajo de un kilómetro, los traslados entre uno y otro, significan un esfuerzo considerable pues hay que cubrirlos a pie. Los negocios que proveen diferentes servicios ocupan inmuebles que no fueron diseñados, desde el principio, con tal fin. Por ejemplo, se puede citar el caso de casonas que han sido adaptadas para abrir negocios que atienden un mercado potencial de clientes nacionales y extranjeros. Algunos trabajan legalmente, otros funcionan bajo la clandestinidad. Se comprobó que los turistas extranjeros llegan a estos lugares en forma notoria.

En esta parte de la ciudad se emplazan saunas; urinarios públicos; bares y cafés; discotecas y lugares de alojamiento temporal. Algunos funcionan sólo ciertos días de la semana, en especial de viernes a domingo. Asimismo, en esta zona hay una cantidad importante de "padrotes" (nombre local de los hombres que ofrecen servicios sexuales), en particular en la calle que bordea el costado norte del edificio de la Biblioteca Central, y hacia el oeste de la misma, además de la Cuarta Avenida, entre las calles 6 y 11. Aunque los hay de origen guatemalteco, en este sector predominan jóvenes y adultos hondureños que trabajan ahí. Muchos de ellos son migrantes que se encuentran temporalmente en Guatemala, en un viaje terrestre más largo que tiene a Estados Unidos como destino final. Los padrotes con apariencia europea ocupan la calle 8; en las calles 6 y 7 se ubican aquellos de biotipo ladino o indígena. Los precios de sus servicios oscilan entre los 3 y 25 dólares de Estados Unidos, cantidad superior al salario mínimo nacional que es de 1.2 dólares la hora (Gobierno de Guatemala, 2014).

Cerrito del Carmen. Da nombre al lugar donde se asienta la ciudad, valle de la Ermita del Carmen. Su topografía permitió, durante mucho tiempo, el encuentro de personas, tanto en las laderas del cerrito como en negocios de alojamiento, próximos a este, que florecieron consecuentemente y que aún siguen en funciones. De siempre ha sido un lugar de prostitución femenina y masculina. La zona de concurrencia de muchachos jóvenes (que llegan ahí para prostituirse) y posibles clientes (en general, hombres maduros) se encuentra alrededor de la iglesia, en la cima del cerro, a la que se 
Álvaro Sánchez-Crispín, Gerardo Mollinedo-Beltrán. Geographical dimension of

MSM socialization spaces in Guatemala City and its relationship with tourism

DOI: http://dx.doi.org/10.15359/rgac.1-56.6

accede tanto a pie como en automóvil. A pesar de los anuncios contra actos inmorales, colocados en la parte posterior del inmueble, y de la postura hostil del mando eclesiástico, la socialización entre HSH está presente desde las primeras horas del día hasta que el cierre de las rejas que circundan el cerro. Las personas que acuden a este lugar de la ciudad son de todas edades y biotipos; los turistas extranjeros raramente llegan aquí.

SO36. Es un club de encuentro. Está hacia la periferia de la Zona 1, en los límites con la Zona 2. Se trata de un lugar oscuro, en dos niveles, aunque en realidad es sólo uno el que está abierto a la clientela. Es un negocio que exhibe todos los permisos correspondientes, otorgados por la municipalidad y permite la propaganda de diversas organizaciones no-gubernamentales y de salubridad. El costo de la entrada es de poco menos de 5 dólares de Estados Unidos. La clientela es variada y proviene, fundamentalmente, de sectores que pertenecen a la zona metropolitana de la Ciudad de Guatemala, aunque también se produce la llegada de algunos turistas extranjeros. Tiene un horario restringido hasta las primeras horas de la noche.

Sauna Urbanos. Su emplazamiento está inmerso en un escenario repleto de hoteles de paso, ubicados en la $3 \mathrm{~A}$ calle, de difícil hallazgo en la trama urbana. Esta especialización del uso del suelo se expande varias cuadras paralelas hacia el norte, donde hay una gran cantidad de pequeños hoteles con clientela heterosexual. Su vecino en la acera opuesta es el Hotel del Norte, que se explica líneas abajo. Esta calle está considerada como rosa ya que, por lo menos, cinco hoteles ubicados sobre la misma reciben a HSH, en forma abierta o velada. Este sauna tiene un equipamiento restaurado, aunque las dimensiones del predio en el que se encuentra son relativamente pequeñas. De hecho, comparte el inmueble con el hotel Happy Rooster, que ocupa el piso superior; ambos son propiedad del mismo empresario que también ofrece servicio de taxis gay-friendly y de masajes, que se contratan vía internet. El costo de la entrada es de entre 4 y 12 dólares de Estados Unidos, en función del día de la semana; más caro los jueves y los sábados. Al interior del establecimiento se ofrecen servicios, como masajes terapéuticos (20 dólares, por hora), y completos (50 dólares, la hora). Esto está asociado con la demanda hecha tanto por clientes nacionales como por turistas extranjeros, que frecuentan el lugar. Estos últimos 
Álvaro Sánchez-Crispin, Gerardo Mollinedo-Beltrán. Dimensión geográfica de los lugares de socialización entre HSH en la Ciudad de Guatemala y su vinculación con el turismo

DOI: http://dx.doi.org/10.15359/rgac.1-56.6

llegan a Urbanos en mayor cantidad durante las temporadas de fin de año y Semana Santa, procedentes de Estados Unidos y Europa.

Hotel del Norte. Con solo quince habitaciones, es un alojamiento localizado frente al sauna Urbanos que, durante el fin de semana, solo acepta hombres. El precio de alquiler de una habitación es cercano a 6 dólares de Estados Unidos, por una estancia de unas horas; también, se puede acceder y permanecer en los pasillos por un pago de 4 dólares. En parte, este lugar se utiliza para pasar la noche, una vez que se regresa de bares y discotecas de la Zona 1, ya que muchos de sus clientes viven en la periferia de la zona metropolitana de la Ciudad de Guatemala. Su clientela es básicamente joven, y acude en grupos al hotel. Las duchas son colectivas, y esto añade un mayor grado de atracción al lugar. La clientela es básicamente guatemalteca.

Plaza Central y Parque Centenario. Ya desde los años ochenta, Whitman (1980), consideraba que una proporción significativa de la socialización entre HSH, en la Ciudad de Guatemala, ocurría en o alrededor de la Plaza Central. Es un espacio de interacción muy amplio que incluye toda la superficie frente al Palacio Nacional. La dinámica se concreta a un contacto primario para, después, ir a alguno de los varios lugares que existen en la Zona1. La esquina noreste de la plaza y las proximidades de los baños de la Concha Acústica se distinguen por la presencia de hombres que ofrecen sus servicios, tanto guatemaltecos como hondureños y salvadoreños. Este espacio abierto funciona como lugar de encuentro para $\mathrm{HSH}$, en forma velada; justo ahí, el gobierno municipal organiza frecuentemente ferias, conciertos y campañas, lo que hace menos evidente su presencia y la verdadera finalidad con la que llegan a este espacio público.

Baños de la Concha Acústica. Se trata de un lugar altamente concurrido, durante el tiempo que permanece abierto ( 8 a 20 horas), ubicado en el límite oeste del Parque Centenario. Las bancas localizadas en la proximidad de los baños se encuentran ocupadas por jóvenes y personas de edad madura que esperan socializar entre sí. Al interior de los baños la vigilancia es mínima, y la entrada y salida de personas es numerosa. Aquí llegan hombres de todas las zonas de la ciudad, guatemaltecos del interior 
Álvaro Sánchez-Crispín, Gerardo Mollinedo-Beltrán. Geographical dimension of

MSM socialization spaces in Guatemala City and its relationship with tourism

DOI: http://dx.doi.org/10.15359/rgac.1-56.6

y extranjeros. En razón de lo investigado en internet, este es uno de los sitios de cruising más importantes de la capital del país, tanto por el mínimo precio para acceder a las instalaciones (15 centavos de dólar de Estados Unidos) como por la conectividad del lugar, al estar emplazado en el centro mismo de la ciudad y ligado a través de diversas rutas de transporte colectivo, en particular por tener acceso a una de las paradas del sistema Transmetro, a escasos metros de distancia. También, se observó que los hombres que ofrecen sus servicios sexuales transitan por un circuito que incluye la Plaza Central, la Catedral Metropolitana y la Concha Acústica. Los aseadores de calzado y los vendedores informales de la plaza saben de la dinámica social que aquí ocurre; según su opinión, esto se produce porque la Ciudad de Guatemala es liberal y porque llegan hombres de todos lados, con diferentes gustos.

Sexta Avenida. Originalmente, esta era la Calle Real. Convertida ahora en calle peatonal, siempre ha sido un eje de actividad comercial y de servicios para toda la capital guatemalteca. Cuenta con una diversidad de negocios: cafés, restaurantes, tiendas y plazas comerciales. En la actualidad, desde su intersección con la Plaza Central hasta la calle 18, se utiliza como lugar de cruising o ligue. Aunque el término no se refiere exclusivamente a lo anterior, sextear es un verbo usado en la capital guatemalteca para indicar el paseo y tránsito por esta avenida. Aquí se encuentran dos establecimientos íconos de la socialización gay local: el restaurante Burger King, en el cruce de la Sexta Avenida y la calle 10, en particular en el piso superior, desde donde se tiene una vista privilegiada sobre la peatonal; y el restaurante McDonalds de la Sexta Avenida, entre las calles 10 y 11, tanto en la sección contigua a la peatonal como en el segundo nivel del inmueble. Como en otros lugares de este tipo, ambos restaurantes no son evidentes ante los ojos de la mayor parte de las personas, pero son totalmente identificables por los HSH que sextean. Tener acceso a estos sitios no cuesta y toda clase de hombres puede llegar a ellos debido a su ubicación céntrica, a lo que se aúna el hecho de permanecer abiertos hasta relativamente tarde (cierran cerca de las 22 horas). Por estas razones, son considerados lugares preferenciales de socialización entre HSH en la ciudad. 
Álvaro Sánchez-Crispín, Gerardo Mollinedo-Beltrán. Dimensión geográfica de los lugares de socialización entre HSH en la Ciudad de Guatemala y su vinculación con el turismo

DOI: http://dx.doi.org/10.15359/rgac.1-56.6

Cyber Café IT. Es el más antiguo de los varios cafés internet del centro de la capital guatemalteca que sirven, doblemente, como lugares de socialización entre HSH. Se ubica en el segundo piso del edificio más alto del centro de la ciudad, sobre la acera poniente de la Séptima Avenida, casi al llegar a la Plaza Central; a diferencia de otros negocios que atienden una clientela de HSH, tiene dibujada una modesta bandera arco iris en la pared externa del establecimiento. El acceso es complicado porque implica subir varios juegos de escaleras, tanto para llegar al café como al interior de este. Aunque, oficialmente, el servicio proveído es el uso de computadoras, en realidad los clientes aprovechan la oscuridad del lugar para tener sexo. Su ubicación privilegiada lo hace uno de los sitios más concurridos por $\mathrm{HSH}$, aunque el precio de entrada sea el equivalente de un dólar y medio. La clientela es muy diversa e incluye tanto $\mathrm{HSH}$ nacionales como extranjeros. Durante los fines de semana, tiene un horario extendido hasta las 9 pm, más tarde que cualquier otro negocio de su tipo en la Zona 1.

Laberinto. Café internet, abierto a finales de 2013. Está en las cercanías de la terminal Plaza Barrios del Transmetro, sobre la Novena Avenida, a la altura de la calle 19, lo que facilita su accesibilidad desde diferentes puntos de la capital. Aunque el servicio ofrecido es el acceso a computadoras, en realidad el negocio tiene una sección anexa a la que se puede tener ingreso por un pago de cerca de 2 dólares. Está concurrido por una diversidad de HSH nacionales, y funciona en horarios que se extienden sólo hasta las primeras horas de la noche.

Cyber Place. Este negocio se ubica en la Cuarta Avenida y calle 11. Es un café internet que ocupa un inmueble de dos pisos, con instalaciones deterioradas; el superior es el más buscado por HSH pues tiene una superficie muy amplia y obscura; además, en este piso hay un espacio utilizado para encuentros físicos. El precio de entrada es de 1.5 dólares por hora. La clientela que llega es diversa y vive tanto en lugares de la propia capital como de la zona metropolitana (Mixco, Villanueva, Petapa). Es un sitio notoriamente concurrido por una clientela joven, con poca afluencia de turistas extranjeros, cercano al área de prostitución masculina de la Cuarta Avenida. 
Álvaro Sánchez-Crispin, Gerardo Mollinedo-Beltrán. Geographical dimension of

MSM socialization spaces in Guatemala City and its relationship with tourism

DOI: http://dx.doi.org/10.15359/rgac.1-56.6

Black and White. Bar-discoteca alojado, como otros negocios que atienden $\mathrm{HSH}$ en el centro de esta ciudad, en una casa antigua, de grandes dimensiones y acondicionada para los servicios que ahí ahora se proveen. Se ubica en la calle 11. El costo de la entrada es de alrededor de 6 dólares. La clientela es diversa por biotipo y clase socio-económica. Es uno de los espacios de socialización entre HSH más frecuentados de la capital. La ley seca imperante en la Ciudad de Guatemala hace que este sitio, y la mayoría de los otros, cierren sus puertas a la $1 \mathrm{am}$, lo que obliga a abrir cerca de las $8 \mathrm{pm}$, un horario distinto en comparación con lo que ocurre en otras ciudades de América Latina. El establecimiento se compone de dos bares, una pista de baile (sobre lo que antes fuera el patio interno de la casa), una sala de televisor y un cuarto obscuro. Como en otros lugares semejantes de esta ciudad, la entrada a travestíes está prohibida y la cantidad de clientes mujeres es mínima.

K1. Es promocionado como un pub, estilo inglés. Se trata de una gran casona del Centro Histórico, propiedad del mismo dueño del Hostal y del Café de la Hermita (sitios de socialización entre HSH ya cerrados), ubicado sobre la Tercera Avenida, cerca de la calle 6. En las instalaciones hay espacios muy grandes para bailar y un área abierta destinada al uso de los fumadores. No se cobra la entrada y el precio de las bebidas es de alrededor de un par de dólares. Hay venta de ropa y accesorios. Solo abre dos días por semana (viernes y sábado; se arguye que esto es así porque la demanda de la población local no es tan alta como para hacer rentable su apertura toda la semana) y se ciñe a los horarios de la ley seca vigente. Como ocurre en otros sitios de socialización de la capital guatemalteca, una vez producido el encuentro entre $\mathrm{HSH}$, estos se dirigen a otros lugares, que permanecen abiertos más allá de la $1 \mathrm{am}$, o a fiestas privadas. Este lugar es frecuentado por turistas extranjeros, estadounidenses y europeos, tanto por el tipo de ambientación existente como por la calidad de las bebidas y la posibilidad de socializar con hombres guatemaltecos.

Ephebus Le Palais Rouge. Ubicada sobre la calle 4, en las proximidades de la Quinta Avenida, es una discoteca que admite la entrada de travestíes, una de las pocas en hacerlo. Solo abre los sábados y domingos, y no se rige por la ley seca que impera en la ciudad. La entrada es de cerca de 8 
Álvaro Sánchez-Crispín, Gerardo Mollinedo-Beltrán. Dimensión geográfica de los lugares de socialización entre HSH en la Ciudad de Guatemala y su vinculación con el turismo

DOI: http://dx.doi.org/10.15359/rgac.1-56.6

dólares, con derecho a dos bebidas. A semejanza de otros establecimientos para HSH, ubicados en el centro de la ciudad, ocupa una gran casona modificada, con un patio trasero muy amplio. La clientela es variada en edad, estrato socio-económico y etnia. Se suelen realizar concursos tanto de strippers masculinos como de travestíes. Algunas empresas locales financian espectáculos, sobre todo en época de vacaciones (Semana Santa, fin de año). Al no funcionar bajo el esquema de la ley seca, la afluencia de clientes se incrementa después de la 1am. Los turistas extranjeros llegan en cantidad considerable a este establecimiento, dada su ubicación privilegiada y la propaganda que se hace a este negocio, tanto en forma impresa como digital.

El Encuentro. Sobre la Quinta Avenida, entre las calles 10 y 11, este restaurante-bar, alojado en una gran casona, pasa inadvertido a los ojos del público en general. Como bar, abre un par de días a la semana (viernes y sábado) y permanece abierto hasta las 21 horas. A lo largo de los días laborables, funciona como restaurante, con comida típica guatemalteca. La clientela es de hombres maduros. No obstante su ubicación central y accesible, frente a una estación de Transmetro, es un sitio de socialización poco concurrido.

Bar $D \& N$. Se encuentra sobre la calle 11 calle y la Sexta Avenida A. La mayoría de los clientes que acuden a este sitio son locales. Es un punto de referencia para ir a otros lugares conforme la noche avanza. Hay todo género de parroquianos, hombres, mujeres, travestis. La música es estridente, lo que impide la conversación entre personas, situación desventajosa cuando se trata de socializar con extraños. El acceso es libre y el precio de las bebidas módico. La llegada de turistas extranjeros no es frecuente.

Baños El Padrino (antes El Trébol). Establecimiento de baños de vapor, con acción velada, aunque la clientela en su casi totalidad es gay. Ocupa un edificio pequeño de dos niveles, en una esquina de la periferia de la Zona 1 (calle 20 cerca de la 12 Avenida), colindante con las antiguas vías del ferrocarril, área de prostitución femenina, día y noche. A diferencia de otros negocios semejantes, estos funcionan solo unos días a la semana, en horario restringido de 9 a 17 horas. La clientela es mayoritariamente local, de apariencia masculina y de edad madura. El costo de la entrada es 
Álvaro Sánchez-Crispín, Gerardo Mollinedo-Beltrán. Geographical dimension of

MSM socialization spaces in Guatemala City and its relationship with tourism

DOI: http://dx.doi.org/10.15359/rgac.1-56.6

de siete dólares de Estados Unidos. Su ubicación periférica dentro de la Zona 1 los hace inaccesibles para los turistas, además de que es vecina de barrios considerados inseguros. A fines de 2013, estos baños fueron remodelados y su razón social cambió a la de baños El Padrino; aparentemente, ahora no acogen una clientela de HSH.

Zona 2. Esta es un área insegura de la capital de Guatemala, donde no hay muchas facilidades o recursos para el turismo. Sin embargo, en el límite con la Zona 1, sobre la Séptima Avenida, se encuentra uno de los saunas más concurridos, tanto por clientela guatemalteca como extranjera, muy publicitado en los blogs específicos sobre lugares gays en la Ciudad de Guatemala, Dansei.

Dansei. Se trata de una antigua casa renovada, en un solo nivel, que dispone de jacuzzi, sauna húmedo, sauna seco, sala de televisión, zona de regaderas, cafetería y área de recepción y cambiado. Es uno de los sitios más frecuentados en la Ciudad de Guatemala a pesar de que los precios de entrada son elevados (más de 12 dólares de Estados Unidos, el día más caro), en comparación con el salario de un guatemalteco promedio. Hay fiestas temáticas y permanece abierto entre las 12 y las 21 horas. No obstante ser vecino de una zona peligrosa, la transportación desde el sauna, una vez cerrado este, es fácil pues existe el enlace con un servicio de taxis, que trabaja ex profeso con el negocio. La clientela es variada e incluye turistas internacionales de distinta procedencia (Estados Unidos, México, El Salvador, Costa Rica, Panamá, Argentina, Alemania e Italia). También, llegan hombres desde lugares cercanos a la capital como Mixco, Amatitlán y Villanueva.

Zona 4. Desde 2006, el área llamada Cuatro Grados Norte, fue promovida por el gobierno local, como un destino para los turistas que buscan vida nocturna, en un ambiente relativamente seguro. Ahí, se ubican dos de las discotecas más concurridas por HSH.

G Lounge. Está ubicada en contraesquina del hotel Plaza Maya. Funciona solo un par de días a la semana: miércoles y jueves. Coordina sus actividades con Genetic Majestic Club, negocio cercano que abre solo los 
Álvaro Sánchez-Crispín, Gerardo Mollinedo-Beltrán. Dimensión geográfica de los lugares de socialización entre HSH en la Ciudad de Guatemala y su vinculación con el turismo

DOI: http://dx.doi.org/10.15359/rgac.1-56.6

viernes y sábados; y es parte de la empresa Clubs Majestic Guatemala, al que también pertenecen la discoteca Ephebus La Palais Rouge y el sauna GWet. Ocupa un solo nivel, cuya característica peculiar es tener un túnel donde se puede bailar. La clientela es tanto nacional como extranjera; los turistas, provenientes de distintos países (El Salvador, México, Costa Rica y Estados Unidos) se alojan en hoteles de la Zona 4, como El Conquistador y el Plaza Maya, y aún los que se hospedan en las zonas 9 y 10, pueden acceder fácilmente a este negocio. El precio de entrada es superior a 13 dólares por persona, con derecho a bebida ilimitada. Se permite el ingreso a mujeres y personas travestidas. Esta discoteca es muy concurrida porque permanece, oficialmente, abierta hasta la una de la mañana. Hay una dinámica que la enlaza con hoteles modestos, cercanos, donde se alojan algunos de sus clientes que viven en lugares relativamente alejados de la capital guatemalteca (Escuintla, Antigua). Las leyes municipales prohíben fumar dentro de locales cerrados, esto implica que los clientes de $G$ Lounge deben hacerlo en la calle donde se emplaza la discoteca, lo que la visibiliza. Como en otros negocios similares, esta discoteca ofrece servicios de taxis seguros.

Genetic Majestic Club. Se considera la discoteca gay más grande de la ciudad. Es un sitio donde, además, se organizan concursos, espectáculos y promociones. Constituye el núcleo de la empresa Clubs Majestic Guatemala, mencionada líneas arriba. Es un inmueble de tres niveles, muy cercano a otros negocios del ramo, orientados a una clientela heterosexual, en contra esquina de la tienda CEMACO. El precio de entrada es, como en el G Lounge, cercano a los 13 dólares de Estados Unidos y la llegada de turistas extranjeros a este lugar es notoria. Sus instalaciones son modernas y, aunque el horario oficial de funcionamiento es hasta la una de la mañana, la discoteca permanece abierta hasta más tarde y esto la hace más atractiva.

Zona 6. Se trata de un sector de la ciudad de poco interés para el turismo. En las cercanías de la Parroquia de la Santa Cruz del Milagro, sobre la 15 Avenida, se encuentra un sitio que, en octubre de 2013, se declaró abiertamente dirigido a HSH, su razón social es Spa Oasis La Parroquia. 
Álvaro Sánchez-Crispín, Gerardo Mollinedo-Beltrán. Geographical dimension of

MSM socialization spaces in Guatemala City and its relationship with tourism

DOI: http://dx.doi.org/10.15359/rgac.1-56.6

Spa Oasis La Parroquia. Pertenece al mismo dueño de los baños Spa Oasis Altamira, de la Zona 7. El inmueble que ocupa data de, por lo menos, hace 30 años; sus servicios originalmente estaban dirigidos a los habitantes de los barrios próximos. Es un negocio muy amplio, ubicado a mitad de cuadra, con entrada discreta. Sus instalaciones fueron renovadas y ampliadas a mediados de 2013. El spa tiene un problema de accesibilidad pues su ubicación, al norte de la avenida Martí, la separa de las áreas vecinas debido a que hay pocos puntos para cruzarla, tanto a pie como en vehículo, y a la existencia de una barrera de contención al centro de la avenida. La clientela es básicamente de hombres maduros. El costo de entrada es cercano a 8 dólares por persona. Además de las instalaciones de baños de vapor, hay regaderas, sala de descanso, zona de restauración, área de masajes y cuarto obscuro. Algunos fines de semana estos baños permanecen abiertos hasta altas horas de la noche, aunque en general la hora de cierre es las $9 \mathrm{pm}$. La llegada de turistas extranjeros (estadounidenses, europeos y de naciones centroamericanas vecinas de Guatemala) es frecuente. Muchos de los clientes nacionales viajan varios kilómetros para llegar ahí; la razón esgrimida, por algunos informantes, es que la lejanía les asegura no ser vistos o descubiertos por gente que los conozca.

Zona 7. En ella se encuentran dos establecimientos orientados a una clientela nacional de $\mathrm{HSH}$.

Spa Oasis Altamira. Pertenece a la misma cadena del sauna ubicado en la Zona 6. Es una construcción nueva, en dos niveles. Se ubica cerca de otros baños famosos, los Marrio's. Es un lugar al que asiste una clientela joven, con mayores pretensiones que la que acude a otros establecimientos de este tipo. Hay un baño de vapor seco y otro húmedo; masajes y una sala de estar; asimismo, se ofrecen bebidas y colaciones gratis. También, está abierto hasta relativamente tarde (20-21 horas), todos los días, lo que lo hace singular en el ambiente capitalino. Su accesibilidad es un factor de ventaja, pues se ubica sobre la 23 Avenida, a unos pasos de la calzada Roosevelt, en el mismo flanco en el que se encuentran los Marrio's. Aquí llegan algunos turistas extranjeros (de América del Norte y Europa occidental). El precio de entrada es de 11 dólares. 
Álvaro Sánchez-Crispin, Gerardo Mollinedo-Beltrán. Dimensión geográfica de los lugares de socialización entre HSH en la Ciudad de Guatemala y su vinculación con el turismo

DOI: http://dx.doi.org/10.15359/rgac.1-56.6

Baños turcos Marrio's (en alusión a Marriot's, la cadena hotelera internacional). Ocupa un edificio de los años setenta, con letrero muy visible, próximo al cruce de la Avenida Roosevelt y la 19 Avenida. Aunque no es un establecimiento abiertamente gay, la mayoría de los clientes son HSH que viven en el perímetro próximo de los baños. Casi no llegan extranjeros, pero sí guatemaltecos del interior quienes aprovechan la ubicación del establecimiento cercana a la Carretera Panamericana (Avenida Roosevelt), con conexiones relativamente fáciles hacia el centro y occidente de Guatemala, en particular con Mixco y Antigua. Se constituye por dos niveles, en el inferior está la zona de ingreso, un restaurante, una zona para masaje y dos baños de vapor (húmedo y seco); en la parte alta, se localiza el solarium, un gimnasio y un jacuzzi en mal estado. Funciona todos los días del año y las horas de cierre son relativamente tarde (20 a 21 horas). La entrada es de poco menos de 10 dólares de Estados Unidos. La llegada de turistas extranjeros es reducida porque la zona donde están ubicados es insegura.

Zona 9. Dispone de infraestructura urbana aceptable y goza de calidad alta en los servicios municipales proveídos. Aún así, es hasta 2014 que se abre, en esta parte de la ciudad, un sitio de socialización entre HSH. Se trata de GWet, sauna próximo a los grandes hoteles y edificios de la zona 10, solo separado de estos por la avenida Reforma donde se encuentra emplazado, a la altura de la calle 12 .

Sauna GWet. Ocupa una vieja construcción, remodelada, que albergó oficinas. Es un inmueble de tres niveles, con amplio espacio al frente para estacionamiento. En realidad es en un solo piso donde se encuentran todas las facilidades del establecimiento. Es un baño de vapor, equipado y funcionando en forma correcta (a diferencia de lo que ocurre en Dansei o Urbanos). La entrada por persona es cercana a los 13 dólares de Estados Unidos; esto hace que sus servicios estén dirigidos a guatemaltecos de alto poder adquisitivo y turistas extranjeros que se alojan en los hoteles de la zona 10, como el Holiday Inn, Radisson, Clarion Suites, Intercontinental Guatemala y Mercure. Como ocurre en los otros establecimientos de esta ciudad, no hay anuncio alusivo y es difícil llegar a él, ya que pasa inadvertido ante los ojos de la población en general. La clientela guatemalteca, 
Álvaro Sánchez-Crispín, Gerardo Mollinedo-Beltrán. Geographical dimension of

MSM socialization spaces in Guatemala City and its relationship with tourism

DOI: http://dx.doi.org/10.15359/rgac.1-56.6

que llega en automóvil, al estacionar sus vehículos, cubre las placas de los mismos para no ser detectados. Al interior de estos baños se encuentra colgado un código de conducta que prohíbe actos inmorales. Es importante indicar que este negocio forma parte de la empresa Clubs Majestic Guatemala, de capital nacional ya mencionado. Su ubicación vecina a la zona 10 le ha asegurado la demanda constante de turistas extranjeros que llegan a este negocio, en especial en época de vacaciones.

Zona 10. Es una de las áreas residenciales de mayor poder adquisitivo en la ciudad, y asiento de diversos hoteles y sedes de empresas nacionales y extranjeras, por tanto, bien aquilatada por personas de negocios, algunos de los cuales pueden querer visitar distintos sitios de socialización entre HSH. A pesar de que hace unos años en esta zona existían cerca de 20 manzanas con comercios y servicios, de categoría superior, orientados al turismo extranjero (particularmente estadounidense), esto ha venido a menos desde 2012, cuando hubo violencia muy marcada en sus calles. En esta zona existieron los baños sauna Il Coliseum y Spa Marinos, que funcionaron en forma legal, el primero, durante casi diez años y, el segundo, durante dos. En la actualidad, solo quedan los retretes del Centro Comercial Los Próceres como lugar de socialización de HSH.

Retretes del Centro Comercial Los Próceres. Ubicados tanto en el área de comida rápida como al interior de la tienda ancla SIMAN (tienda de departamentos de origen salvadoreño). No se cobra la entrada a estos servicios, lo que hace que la clientela de ambos lugares sea amplia (de distintas etnias y estratos socio-económicos), con actividad intensa durante todo el día. No son un lugar para tener relaciones físicas sino para contactar a alguien, y después dirigirse a otros sitios. No obstante, la presencia numerosa de turistas en la zona, su visita a estos retretes es poco frecuente.

Zona 11. Es una parte de la ciudad de fácil accesibilidad. Los lugares de socialización entre HSH se limitan a los retretes ubicados en el Centro Comercial Tikal Futura, en el centro comercial Wal-Mart de esta zona (no visitados en el desarrollo de esta investigación) y en el cruce El Trébol. 
Álvaro Sánchez-Crispin, Gerardo Mollinedo-Beltrán. Dimensión geográfica de los lugares de socialización entre HSH en la Ciudad de Guatemala y su vinculación con el turismo

DOI: http://dx.doi.org/10.15359/rgac.1-56.6

Retretes del Centro Comercial Tikal Futura. Como en el caso de los que están ubicados en el Centro Comercial Los Próceres, se emplazan en la zona de comida rápida. Son frecuentados, en su mayoría, por guatemaltecos que viven en la propia capital, de diversas edades y etnias, y distintos estratos sociales y económicos. El acceso a este lugar se ve ampliamente favorecido por la cercanía de la Avenida Roosevelt, que permite enlaces rápidos y frecuentes con muchas partes de la ciudad, y allende esta. Otra ventaja es que no se paga por entrar a los servicios.

Retretes de El Trébol. Entre todos los sitios de socialización entre HSH de la Ciudad de Guatemala, este es el más sórdido. Las instalaciones son mínimas pero muy concurridas al estar próximos a una estación de transbordo del Transmetro y del cruce de la Calzada Aguilar Batres y el boulevard Liberación. No hay costo de entrada y están siempre abiertos. La afluencia de HSH es nutrida e incluye una amplia diversidad de personas; los turistas extranjeros raramente llegan a este lugar.

Periferia de la Ciudad de Guatemala. Se hallaron dos sitios de socialización entre HSH fuera de la trama urbana central: los retretes de la estación Centra Sur del Transmetro y la Finca Cabañas, ubicada en San Miguel Petapa.

Retretes Centra Sur. Lugar muy frecuentado pues se encuentra en el centro comercial del término sur de la línea del Transmetro. La llegada de $\mathrm{HSH}$ a estos servicios es numerosa; se trata de personas que viven en la periferia de la ciudad y que, al ir o regresar de sus trabajos, utilizan estos retretes, cuyo costo es de 10 centavos de dólar estadounidense. Por su distancia con el Centro Histórico (más de 15 kilómetros), a este sitio no llegan los turistas.

Finca Cabañas. Se promueve como lugar de turismo gay. En particular, los clientes a los que está dirigido este negocio son salvadoreños. Se argumenta que en San Salvador hay bares gays pero nada más. Este es un sitio donde se puede pernoctar, además de contar con facilidades como jacuzzi, spa, restaurante y áreas comunes. Se encuentra relativamente aislado, en una calle sin salida, y el acceso es difícil porque no hay pavimento 
Álvaro Sánchez-Crispín, Gerardo Mollinedo-Beltrán. Geographical dimension of

MSM socialization spaces in Guatemala City and its relationship with tourism

DOI: http://dx.doi.org/10.15359/rgac.1-56.6

$\mathrm{y}$, en tiempo de lluvias, el tránsito es complicado. Para poder llegar hay que hacerlo en auto o en viaje organizado (como los tours que se originan en San Salvador). Es un lugar especial, no solo por el hecho del cobro de entrada, de casi 18 dólares de Estados Unidos por persona, sino por la inaccesibilidad de sus instalaciones.

\section{Discusión de resultados}

A partir del proceso de investigación realizado, se encontraron 33 lugares de socialización entre $\mathrm{HSH}$, en la capital guatemalteca, un número relativamente reducido para su tamaño poblacional. Muchos de estos lugares son poco visibles y funcionan en forma encubierta, lo que les hace vulnerables a ser cerrados en cualquier momento, en razón del ambiente político, o de otra naturaleza, que prevalezca. Debido a los problemas de inseguridad que enfrenta la ciudad, los HSH acuden más a sitios cerrados para socializar (saunas, cibercafés, discotecas); el hacerlo en lugares abiertos los arriesga, tanto a peligros físicos como de índole personal, ya que podrían ser reconocidos o descubiertos.

Hay una concentración de estos sitios en la Zona 1 (más de dos tercios del total), donde también se ubican importantes recursos e infraestructura para el turismo; se conforma, así, el núcleo medular de lugares de socialización entre HSH en esta urbe, extendido por varias manzanas cuyos límites son la calle 3 al norte, la calle 14 al sur, la Primera Avenida al oeste y la Novena Avenida al este (figura 1). Aquí se encuentran los cuatro tipos de lugares identificados en este estudio, diferenciados por sus rangos de accesibilidad, antigüedad, costo, promoción e instalaciones, con un ligero predominio de sitios accesibles, mayormente gratuitos o de costo bajo, y cuya promoción es prácticamente inexistente que, a su vez, son los que prevalecen en la ciudad. Los de mejores instalaciones, con acceso limitado por el costo de entrada relativamente alto, y muy promocionados se encuentran dispersos en el Centro Histórico o Zona 1, muy alejados entre sí. Asimismo, es importante indicar que las calles y áreas de prostitución masculina sólo se hallan en esta parte de la capital y en las proximidades de lugares turísticos importantes como la Plaza Mayor o la Sexta Avenida. Se revela, así, la mayor densidad de lugares de socialización entre HSH del país y, muy probablemente, de América Central. La Zona 1, es escenario de una interacción social amplia por lo que los intereses de turistas y de HSH son coincidentes en esta porción del espacio urbano. 
Álvaro Sánchez-Crispin, Gerardo Mollinedo-Beltrán. Dimensión geográfica de los lugares de socialización entre HSH en la Ciudad de Guatemala y su vinculación con el turismo

DOI: http://dx.doi.org/10.15359/rgac.1-56.6

En el resto de la ciudad, se detectaron lugares que se encuentran dispersos. Al noroeste, sobre la avenida Roosevelt, hay varios sitios de interés para HSH. Aunque su accesibilidad es amplia, por su ubicación no central son más frecuentados por hombres que viven en zonas vecinas que por turistas, son lugares diferenciados por su accesibilidad (al estar dentro de plazas comerciales), poca promoción y costos de entrada bajos. Hacia el sur del Centro Histórico, se encuentran sitios en la zona Cuatro Grados Norte y uno de reciente aparición en la Zona 9; son de alto costo y, por tanto, de acceso restringido, con instalaciones en buen estado. En ellos, la afluencia de turistas (guatemaltecos y extranjeros) es notoria, debido a su proximidad con los hoteles de categoría superior.

Al considerar el alcance geográfico de los lugares estudiados, se advierte la conformación de un hinterland, perfilado sobre la zona metropolitana de la capital guatemalteca y que se extiende hasta Antigua. En un ámbito allende las fronteras nacionales, los sitios aquí revelados ejercen una atracción importante sobre HSH salvadoreños, de status económico alto, que viven en San Salvador. Del mismo modo, se detectó que turistas procedentes de América del Norte, Europa occidental y América Central llegan a la Ciudad de Guatemala (por motivación sexual expresa o no), y visitan varios de los lugares de socialización entre HSH analizados en este trabajo.

\section{Agradecimiento}

Agradecemos a José Alberto Garibay Gómez y Rodrigo Pérez Toledo, de la Universidad Nacional Autónoma de México, su colaboración en este trabajo.

\section{Referencias}

Arboleda, M. (1995) "Social attitudes and sexual variance in Lima" Latin American Male Homosexualities. University of New Mexico Press. Albuquerque, Estados Unidos. pp.100-110

Carrasco, M. (2005) ¿Comunidad homosexual? Gays y lesbianas en Santiago de Chile. Tesis de licenciatura en Psicología comunitaria. Universidad de Chile. Santiago, Chile.

Caplow, T. (1949) "The social ecology of Guatemala City". Social Forces,

28-2. Oxford University Press. pp.113-133 
Álvaro Sánchez-Crispín, Gerardo Mollinedo-Beltrán. Geographical dimension of

MSM socialization spaces in Guatemala City and its relationship with tourism

DOI: http://dx.doi.org/10.15359/rgac.1-56.6

Cicerone (2012) Directorio de Turismo de Centroamérica y del Caribe. Cicerone S.A. Guatemala, Guatemala.

Cifuentes, M. (2008) Expresión gráfica de la dinámica social territorial en Guatemala. Serie: El proceso de urbanización en Guatemala, 1944-2002. Centro de Estudios Urbanos y Regionales. Universidad de San Carlos de Guatemala. Guatemala, Guatemala.

Gellert, G. (1990) "Desarrollo de la estructura especial de la Ciudad de Guatemala: desde su fundación hasta la Revolución de 1944" Anuario de Estudios Centroamericanos. Universidad de Costa Rica. San José, Costa Rica. pp. 31-55

Guasch, O. (1995) La sociedad rosa. Anagrama. Barcelona, España.

Gobierno de Guatemala (2014) Salarios mínimos efectivos del 1 de enero al 31 de diciembre de 2014. Gobierno de Guatemala. Ciudad de Guatemala, Guatemala.

Laguarda, R. (2011) La calle de Amberes: gay street de la Ciudad de México. Centro de Investigaciones Interdisciplinarias en Ciencias y $\mathrm{Hu}-$ manidades. Universidad Nacional Autónoma de México. Ciudad de México, México.

Levine, M. (1979) "Gay ghetto" Gay Men. The Sociology of Male Homosexuality. Harper and Row Publishers. Nueva York, Estados Unidos. pp. 182-204

López, A., Sánchez-Crispín, A. (2004) "Dinámica territorial del deseo queer en Monterrey, México" Ciudades, 62. Revista de la Red Nacional de Investigación Urbana. México, Distrito Federal. ISSN: 0187-8611. pp. 25-33

López, A., van Broeck A. (2013) Turismo y sexo en México. Cuerpos masculinos en venta y experiencias homoeróticas. Una perspectiva multidisciplinaria. Colección para el Siglo XXI. Instituto de Geografía, Universidad Nacional Autónoma de México. México, México.

Mancero, C. (2007). La construcción del movimiento gay y sus manifestaciones sociales, culturales y políticas en la ciudad de Quito. Tesis de maestría en Antropología Social. FLACSO. Quito, Ecuador.

Obando, M. (2003). Homosexualidad: un problema de discriminación social. Tesis de licenciatura en Trabajo Social. Universidad de San Carlos de Guatemala. Ciudad de Guatemala, Guatemala. 
Propin, E. (2003). Teorías y métodos en geografia económica. Universidad Nacional Autónoma de México, Instituto de Geografía. México, México.

Sánchez-Crispín, A., López, A. (1997). “Gay-male places in Mexico City: a geographical interpretation" Queers in Space. Communities, public places and sites of residence. Bay Press. Seattle, Washington. ISBN: 0-941920-44-5. pp. 197-212

Sánchez-Crispín, A., Molliendo, G., Propin, E. (2011). "Estructura territorial del turismo en Guatemala". Investigaciones Geográficas, 78. Instituto de Geografía, Universidad Nacional Autónoma de México. México, México.

Sánchez-Crispín, A., Propin, E. (2010). “Tipología de los núcleos turísticos primarios de América Central" Cuadernos de Turismo, 25. Universidad de Murcia. Murcia, España. pp. 165-184.

Santos, X. (2006). "Territorio e identidad: sexualidades y estrategias espaciales" Lecciones de disidencia. Infoprint. Madrid, España. pp. 45-60

Tripp, C. (1975). The homosexual matrix. A Signet Book. Nueva York, Estados Unidos.

Whitman, F. (1980). The Prehomosexual male child in three diferent socities: the United States, Guatemala and Brazil. Archives of Sexual Behaviour, 9-2. pp. 87-99. 